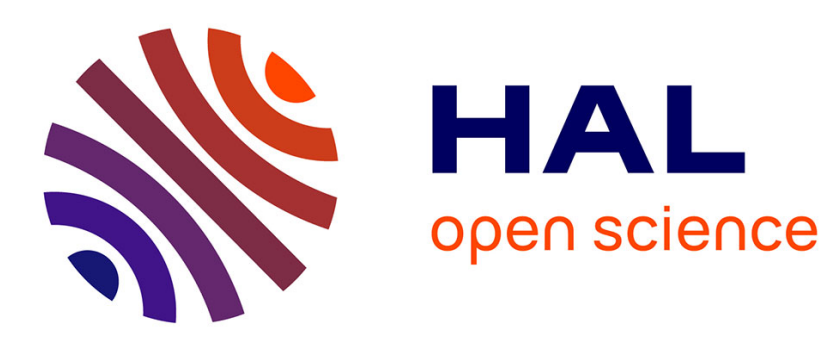

\title{
La participation du refoulement organique et la formation du caractère
}

François Villa

\section{To cite this version:}

François Villa. La participation du refoulement organique et la formation du caractère. Revue Française de Psychanalyse, 2014, 78 (4), pp.978 - 989. 10.3917/rfp.784.0978 . hal-01496255

\section{HAL Id: hal-01496255 \\ https://hal.science/hal-01496255}

Submitted on 27 Mar 2017

HAL is a multi-disciplinary open access archive for the deposit and dissemination of scientific research documents, whether they are published or not. The documents may come from teaching and research institutions in France or abroad, or from public or private research centers.
L'archive ouverte pluridisciplinaire HAL, est destinée au dépôt et à la diffusion de documents scientifiques de niveau recherche, publiés ou non, émanant des établissements d'enseignement et de recherche français ou étrangers, des laboratoires publics ou privés. 


\title{
LA PARTICIPATION DU REFOULEMENT ORGANIQUE À LA FORMATION DU CARACTĖRE
}

\author{
François Villa
}

Presses Universitaires de France | Revue française de psychanalyse

\author{
2014/4 - Vol. 78 \\ pages 978 à 989
}

ISSN 0035-2942

Article disponible en ligne à l'adresse:

http://www.cairn.info/revue-francaise-de-psychanalyse-2014-4-page-978.htm

Pour citer cet article :

Villa François, «La participation du refoulement organique à la formation du caractère »,

Revue française de psychanalyse, 2014/4 Vol. 78, p. 978-989. DOI : 10.3917/rfp.784.0978

Distribution électronique Cairn.info pour Presses Universitaires de France.

(C) Presses Universitaires de France. Tous droits réservés pour tous pays.

La reproduction ou représentation de cet article, notamment par photocopie, n'est autorisée que dans les limites des conditions générales d'utilisation du site ou, le cas échéant, des conditions générales de la licence souscrite par votre établissement. Toute autre reproduction ou représentation, en tout ou partie, sous quelque forme et de quelque manière que ce soit, est interdite sauf accord préalable et écrit de l'éditeur, en dehors des cas prévus par la législation en vigueur en France. II est précisé que son stockage dans une base de données est également interdit. 


\section{Perspectives théoriques}

\section{La participation du refoulement organique à la formation du caractère}

François VILLA*

Je profite de l'invitation à contribuer à ce numéro de la $R f p$ pour apporter un complément à mon essai sur la notion de caractère (Villa, 2009) en précisant la contribution du refoulement organique à la formation du caractère. En reprenant mon travail, j'ai constaté avec étonnement que je n'avais absolument pas pris en compte ce mécanisme et cela alors même que, dans la même période ${ }^{1}$, je commençais à découvrir et à explorer les ressources de l'énigmatique processus freudien du refoulement organique du point de vue des effets du temps sur la vie psychique et de la construction psychique du corps. Je vais donc essayer d'établir la corrélation entre formation du caractère et refoulement organique que je ne fus pas à même de saisir à l'époque.

\section{RÉSORPTION DE L’ÉROGÉNÉITÉ D’UN FONCTIONNEMENT D’ORGANE}

Ce travail s’inscrit également dans la suite de Caractère et érotisme anal et Des transpositions pulsionnelles en particulier dans l'érotisme anal écrits

* Psychanalyste, membre de l'Association Psychanalytique de France et de l'Association Psychanalytique Internationale. Professeur de Psychopathologie, Centre de Recherches Psychanalyse, Médecine et Société (EAD N 3522), Université Paris Diderot - Sorbonne Paris Cité 75013 Paris, France.

1. C’est en effet la même année, 2004, que je publie « Le caractère dans la théorie freudienne : une "sous-espèce de sublimation" dans les transpositions pulsionnelles ", in J. Bouhsira (Dir.), S. Dreyfus (Dir.), A. Fine (Dir.), Caractère(s), Monographies de la revue Française de Psychanalyse, Paris, Puf, p. 11-43 et « À propos de l'ordinaire et extraordinaire détermination humaine à rester en vie », Champ psychosomatique, 35, Éd. L'esprit du temps, p. 103-127. C'est dans ce dernier texte que pour la première fois je m'attaque à éclairer la notion de refoulement organique qui connaitra par la suite d'autres développement recueillis dans mon livre La puissance de vieillir, Paris, Puf, 2010. 
par Freud en 1908 et en 1915. Comme nous allons le montrer la notion de refoulement organique est implicitement présente dans le premier texte cité. Freud y soutient que l'on retrouverait dans l'enfance des personnes qui réunissent à l'âge adulte les trois caractéristiques d'être ordonné, économe et entêté, une période dans leur constitution sexuelle où exista une " accentuation érogènes des plus nettes de la zone anale ». C'est le travail de reconstruction de l'enfance par le traitement psychanalytique qui permet de former par induction cette hypothèse. Car, au présent de la vie de l'adulte, « plus rien de ces faiblesses et singularités ne peut être retrouvé »² et, manifestement, «la zone anale a perdu sa signification érogène ». C'est l'élaboration psychanalytique qui permet de poser que, dans le cours du développement, l'accentuation initiale de l'érogénéité anale a subi un processus psychique de réduction - voire d'extinction qui a abouti à la transposition de l'érotisme anal en la triade de traits de caractère. Pour expliquer ce processus de transposition, Freud convoque les Trois essais sur la théorie sexuelle (1905).

S'il rappelle d'une part «que la pulsion sexuelle de l'être humain est hautement composée, qu'elle naît des contributions de nombreuses composantes et pulsions partielles » et, d'autre part, que l'excitation sexuelle qui a pour source ces " lieux du corps privilégiés qui méritent le nom de "zones érogènes" » joue le rôle essentiel, c'est pour aussitôt indiquer que "les grandeurs d'excitation » qui peuvent se condenser en ces lieux « ne connaissent pas toutes - ni à toutes les époques de la vie - le même destin ». L'un des destins possible de cette excitation est celui qui conduira par la voie des formations réactionnelles vers l'instauration de ce que l'on désigne comme le caractère. Une partie des ces grandeurs d'excitation se verra déviée des buts sexuels et ne participera pas directement à la vie sexuelle ouvrant la possibilité du processus de sublimation. Dans Les trois essais, Freud assimile le mécanisme des formations caractérielles qui sont l'une des sources principales de la formation du caractère à une " sous-espèce de sublimation ». J'ai attiré précédemment ${ }^{3}$ l'attention sur l'importance clinicothéorique de la discussion où Freud, tout en indiquant les points de concordance qui existent entre elles, s'efforce de distinguer la sublimation de ces formations réactionnelles qu'il qualifie de sous-espèce de la sublimation, je ne reviendrai donc pas là-dessus. Dans Caractère et érotisme anal, cette piste est réempruntée sans faire appel, cette fois-ci, à la catégorie de sous-espèce de sublimation :

2. Faiblesses et singularités se manifestaient par l'incontinence fécale permanente ou épisodique, la difficulté à aller sur le pot, la rétention des selles et la manipulation inconvenante des excréments, toutes ces pratiques s'accompagnant d'un gain indéniable de plaisir.

3. Voir le chapitre VI «1905, le caractère : sous-espèce de sublimation, formations réactionnelles », in Villa, 2009, p. 21-29. 
À l'époque de la vie qui peut être qualifiée de «période de latence sexuelle » [...] sont créées dans la vie d'âme - même au prix de ces excitations livrées par des zones érogènes des formations réactionnelles, des contre-puissances, comme la pudeur, le dégoût et la morale, qui s'opposent, tout comme des digues, à la mise en activité ultérieure des pulsions sexuelles. Or comme l'érotisme anal appartient à ces composantes de la pulsion qui, au cours du développement et dans le sens de notre actuelle éducation culturelle, deviennent inemployables à des fins sexuelles, il serait tentant de reconnaître dans ces particularités de caractère - être ordonné, économe et entêté - apparaissant si fréquemment chez les érotistes-anaux de jadis, les résultats immédiats et les plus constants de la sublimation de l'érotisme anal (Freud, 1908b, p. 191).

CHANGER DE CORPS, CHANGER DE CARACTÈRE

Nous croyons volontiers Freud quand il précise à plusieurs reprises que la possibilité d'établir « une corrélation organique » entre les particularités d'un caractère et un comportement d'organe fut une surprise car aucune attente théorique ne l'avait préparé à une telle éventualité. Mais, une fois dépassé l'étonnement, notre auteur n'hésitera pas établir un rapport strictement proportionnel entre la présence de certains traits de caractère et la persistance ou non de l'érotisme anal :

S'il y a quelque chose de factuel à la base des relations affirmées ici entre l'érotisme anal et cette triade de particularités de caractère, on ne pourra guère s'attendre à trouver que le « caractère anal » soit spécialement marqué chez des personnes qui ont conservé dans leur maturité l'aptitude érogène de la zone anale, comme par exemple certains homosexuels. Si je ne m'abuse, l'expérience concorde très bien, la plupart du temps, avec cette conclusion (Freud, 1908b, p. 194).

Le dernier paragraphe du texte s’interrogera sur la possibilité - voire la nécessité - d'étendre cette découverte en se demandant « si d'autres complexes de caractère ne permettent pas de reconnaître qu'ils relèvent des excitations de zones érogènes déterminées » qui ont perdu leur aptitude érogène, celle-ci ayant été « absorbée » dans le processus de transposition de l'érotisme d'organe en traits de caractère. Ce qui nous a incité à opérer le rapprochement avec le refoulement organique et à faire intervenir ce mécanisme dans la formation du caractère, c'est la retrouvaille, dans ce que nous venons de rappeler de ce texte de 1908, de l'essentiel de l'argumentation présente lors de la première présentation par Freud (2006, p. 353-357) de cette notion dans sa lettre à Wilhem Fließ du 14 novembre $1897^{4}$.

4. Cette lettre, écrite donc deux mois à peine après la lettre du 21 septembre 1897 (p. 334-337) où il déclare « je ne crois plus à mes neurotica », propose une nouvelle élaboration de la théorie étiologique des névroses où travail psychique et travail de culture sont étroitement corrélés. 
Mais avant d'en venir à une présentation de la notion de refoulement organique, nous reviendrons sur un fragment de Voltaire concernant le caractère que nous avions cité dans notre essai et où il établissait une analogie entre le caractère et l'instinct en se demandant :

Peut-on changer de caractère ? Oui, si l'on change de corps (...) Tant que [de cet homme] ses nerfs, son sang, sa moelle allongée seront dans le même état, son naturel ne changera pas plus que l'instinct d'un loup et d'une fouine. (Voltaire, 1765)

Nous n'avions à l'époque pas accordée suffisamment d'attention à l'affirmation de Voltaire que, pour changer de caractère, il faudrait changer de corps. Dans un mouvement un peut hâtif, nous n'avions pas pris en considération que le corps ne cesse de changer au cours du temps et qu'il n'est en fait jamais dans le même état. Cela est vrai du corps «biologique » mais l'est tout autant du corps « psychique ». Et, nous soutiendrons que l'incessant travail du refoulement organique et l'insistante poussée qui tend au retour de ce refoulé constituent le jeu de force "par où commence le corps humain $»^{5}$, par où il peut psychiquement se construire, par où il se déconstruit " catastrophiquement ${ }^{6}$ en pouvant ou non emprunter une nouvelle bifurcation, engendrer une nouvelle représentation inconsciente et consciente du corps.

Chacune de ces catastrophes engendre des modifications de l'érogénéité dans l'ensemble du corps et dans chacun des organes, lesquelles sont contemporaines des modifications de l'investissement libidinal dans le moi et de la persévérance narcissique ou non dans tel ou tel érotisme d'organe ${ }^{7}$. Les moments catastrophiques sont toujours des moments critiques pour le monde des représentations et des lieux où, en raison des variations d'amplitude énergétique, des expériences de douleur sont présentes. Ce sont donc nécessairement des moments de désinstauration de la représentation du corps propre et de possible réinstauration d'une nouvelle représentation depuis le moment critique douloureux. Ce sont également des points où peuvent survenir des modifications caractérielles : disparition, transformation ou apparition de certains traits de caractères - faut-il rappeler que le trait de caractère, étant le

5. Je renvoie bien évidemment à Fédida P., Par où commence le corps humain. Retour sur la régression, Paris, Puf, 2000.

6. Le terme de « catastrophe » est à entendre au sens que lui a donné René Thom, il désigne le lieu où une fonction change brusquement de forme.

7. J'emploie persévérance dans le sens que lui donne Freud (1916-1917e, p. 59) : «Lors de la défécation, l'enfant se trouve placé devant une première décision, choisir entre position narcissique et position d'amour d'objet. Ou il cède docilement l'excrément, le "sacrifie" à l'amour, ou il le retient pour la satisfaction auto-érotique, plus tard pour l'affirmation de sa propre volonté. Avec cette dernière décision se constitue le défi (entêtement) qui découle donc d'une persévérance narcissique dans l'érotisme anal ». 
monument commémoratif d'un choix d'objet, d'un mode libidinal de satisfaction, d'un érotisme d'organe auquel il a dû être renoncé, est donc à la fois la trace de la perte à laquelle il a fallu consentir et le refus de cette perte par incorporation ou introjection de ce qui a été perdu.

UN DÉTOURNEMENT DE L’ÉROGÉNÉITÉ DE CERTAINES ZONES DU CORPS

En relisant le début de la lettre qui introduit la notion de refoulement organique, nous sommes saisis fortement par l'impression que cette lettre est, elle-même, une mise en acte in vivo du travail de refoulement qu'elle décrit. Le 14 novembre 1897 est, pour Freud, « un jour dominé par une migraine unilatérale gauche " où il met au monde, "après les horribles contractions de ces dernières semaines, [ce] nouveau morceau de connaissance » qu'il nomme refoulement organique. Cette théorie résulte d'un travail psychique qui s'accomplit en surmontant les affres du doute né de l'abandon des neurotica et dans une lutte pour s'arracher à la domination qu'exerce sur l'esprit un corps migraineux douloureux.

C'est donc la première fois que nous est présentée cette notion qui garde jusqu'à maintenant un caractère d'étrangeté foncière et cela alors même qu'elle jouera un rôle axiomatique tout au long de l'œuvre freudienne. Elle réapparaît à la manière d'une survivance résurgente dans différents travaux. À chacune de ses occurrences, alors qu'elle se révèle comme un des fondements de la construction théorique ${ }^{8}$, elle n'est pourtant pas véritablement développée, mais est toujours présentée comme allant de soi. Il faudra attendre Le malaise dans la culture, (Freud, 1930, note 1, p. 286-287 ; note 2, p. 292-293) pour qu'en soit donnée une explicitation. Remarquons qu'étonnamment, celle-ci sera une reprise quasi à l’identique de la présentation de la lettre du 14 novembre 1897 (dont Freud ne disposait plus à cette époque).

Freud est à la recherche de ce qui « se trouve d'essentiel » derrière le refoulement et il prétend avoir "souvent pressenti que dans le refoulement quelque chose d'organique est à l'œuvre ». Ce quelque chose est d'emblée désigné comme "l'abandon d'anciennes zones sexuelles ». Cette supposition est née chez Freud à partir de l'observation des modifications du rôle et de la place des sensations olfactives qui surviennent dans le cours du développement humain. Notons que la réflexion freudienne s'appuie ici sur les traces de

8. Voir en fin d'article les différentes occurrences de la notion dans l'œuvre freudienne. 
cette modification inscrite dans la langue même. Une expression comme «il porte le nez haut (die Nase hoch tragen) » qui signifie " se croire supérieur, faire le fier, être rempli d'orgueil » porte la trace d'un déplacement du bas vers le haut dans l'espèce humaine résultant du passage à la verticalité qui a eu pour effet une atténuation manifeste de la fonction olfactive : le nez en s'éloignant du sol nous a fait perdre l'intérêt pour certaines sensations qui nous sont même devenues répugnantes. Freud, partant de cet exemple de la zone olfactive, propose de l'étendre à d'autres zones du corps - en premier lieu la région anale. Les sensations propres à ces zones contribuaient, primitivement, à provoquer une « déliaison sexuelle »" mais, à la suite du refoulement organique qu'elles ont subi, ces sensations ne livrent plus de « contribution à la libido ».

La dimension perverse polymorphe de la sexualité infantile se fonde sur le fait que des zones possèdent encore cette érogénéité pleine qui se verra le plus fréquemment drastiquement atténuée dans le cours du développement. Freud découvre ici ce qu'il appellera dans Pour introduire le narcissisme (1914c, p. 227-228) l'érogénéité et qui désigne une propriété générale de l’ensemble du corps et de chacune de ses parties. En écrivant, dans cette lettre, qu' " on doit supposer qu'à l'âge infantile la déliaison sexuelle n'est pas encore aussi localisée qu'elle le sera plus tard, de sorte qu'ici aussi ces zones abandonnées plus tard (peut-être toute la surface du corps aussi) suscitent d'une certaine manière quelque chose qui est analogue à la déliaison sexuelle ultérieure ", l'auteur nous fait entrevoir un état originaire : celui du moi-ça indifférencié où c'est l'ensemble du corps qui est également érogène ${ }^{10}$. Il s'agirait là d'un état du moi-corps antérieur à l'œuvre du refoulement organique qui, par atténuation de l'érogénéité de certaines parties du soma, circonscrira principalement cette propriété aux zones dites érogènes - celles-ci formeront les coordonnées depuis lesquelles se construit psychiquement le corps. L'érogénéité est donc une propriété générale de l'ensemble du corps et de chacune de ses parties mais le droit de disposer de cette propriété peut connaître un accroissement ou un abaissement pour des raisons d'ordre physique ou psychique et peut même entraîner la perte momentanée ou durable, totale ou partielle de l'aptitude érogène. Nous attirerons l'attention sur le fait que perdre, par suite du refoulement, l'aptitude érogène n'est pas perdre pour autant la propriété générale

9. À propos de la déliaison sexuelle, il nous faut concevoir qu'elle engendre en même temps une part de satisfaction et une contribution à la tension sexuelle. Nous retrouvons cette ambigüité à propos de la notion de Lust qui désigne aussi bien la sensation de la tension sexuelle (Ich habe Lust = je voudrais, je ressens l'envie pressante) que celle de la satisfaction (voir Freud, 1905, note 1, p. 151).

10. Sont convoqués ici les modèles élémentaires de représentations que sont d'une part, le modèle de l'œuf, d'autre part, du système nourrisson plus les soins maternels et enfin de l'amibe émettant des pseudopodes (voir Villa F., 2010, chapitre « Le corps sans organe et l'organe hypocondriaque »). 
à tous les organes qu'est l'érogénéité, celle-ci demeure actuelle et en puissance à l'abri du refoulement.

L'examen du destin ultérieur des zones sexuelles abandonnées va donner lieu à un remarquable retour sur la notion d'après-coup ${ }^{11}$. " L'état interne de la libido » que produit la déliaison sexuelle résulte bien sûr, d'une part, des excitations externes et, d'autre part, des excitations internes des zones érogènes mais un autre élément essentiel intervient : les représentations, les traces mnésiques des sensations liées à ces zones. Prendre en considération l'action de ces dernières impliquent nécessairement de faire appel au processus de l'après-coup ${ }^{12}$ et c'est là que Freud rappelle qu'il ne faut pas oublier qu'il y aussi « un après-coup non névrotique sur un mode normal et à partir duquel apparaît la contrainte ». Cet après-coup normal porte bien évidemment aussi sur « les souvenirs concernant les excitations des zones sexuelles abandonnées », mais le retour de ce refoulé a pour conséquence un retournement en son contraire : là, où jadis il y eut déliaison de libido, aujourd'hui, se produit une sensation de déplaisir, « une sensation interne qui est analogue au dégoût dans le cas d'un objet ».

Ce constat conduit Freud à définir le refoulement d'une manière concrètement imagée :

Grossièrement dit, le souvenir pue actuellement, comme l'objet pue dans le présent, et de même que nous détournons dans le dégoût l'organe des sens (la tête, le nez), de même le préconscient et le sens qu'est la conscience se détournent du souvenir. C'est cela le refoulement.

C'est maintenant que nous allons pouvoir revenir à la contribution du refoulement organique à la formation du caractère.

« TOUT CELA APPARAÎT AU PRIX D’UNE SEXUALITÉ DISPARUE (VIRTUELLE)»

Dans cette lettre, ce qui se dévoile c'est la dimension organique qui intervient dans le refoulement et il ne serait pas très audacieux de rajouter particulièrement dans le refoulement originaire. Je poserai axiomatiquement que,

11. N'oublions pas que dans La sexualité dans l'étiologie des névroses, écrit en 1898, un an après l'abandon des neurotica, Freud écrit que « les facteurs principaux sur lesquels s'appuie la théorie des psychonévroses [sont] l'effet d'après-coup, l'état infantile de l'appareil sexué et de l'instrument d'âme ».

12. Je ne résiste pas au plaisir de citer Freud : «Tu connais déjà ce raisonnement : si l’on a irrité un enfant sur ses organes génitaux, il se produit des années plus tard, par l’après-coup du souvenir de cela, une déliaison sexuelle beaucoup plus forte qu’à l'époque, parce que l'appareil prépondérant et le montant de sécrétion ont grandi entre-temps ». 
dans le refoulement originaire, le refoulement organique joue un rôle déterminant et qu'il en est même une part essentielle. Il nous faudra cependant distinguer les deux opérations depuis le facteur temporel. Le refoulement originaire a eu mythiquement lieu jadis en une fois, ses effets demeurent persistants dans les processus psychiques actuels en tant que noyau du refoulé dont la force d'attraction prédispose aux refoulements secondaires qu'ils facilitent. Par contre, le refoulement organique serait agissant, non seulement dans le refoulement originaire mais, aussi, dans tous les refoulements secondaires. Il est un mécanisme qui se reproduit à plusieurs reprises dans l'histoire de la vie psychique, car il accompagne inexorablement tout nouveau refoulement dans la mesure où celui-ci entraîne presque toujours l'abandon ou la modification des modes de satisfaction liés à une certaine zone érogène. Je soulignerais également que le statut de la " motivation » n'est pas le même dans le refoulement originaire et dans les refoulements secondaires. Dans la théorie freudienne, le refoulement secondaire est une action (voire une réaction) résultant d'une prise de position quant à la réalité pulsionnelle. Il est une "décision » prise à la suite d'une activité de proto-jugement. Ce mécanisme de défense relève d'une activité de l'individu qui, pour pouvoir être représentée, nous oblige à concevoir l'existence d'un processus primaire qui échappe à la conscience et dont il n'existe aucun souvenir fixé. Il n'en va pas de même pour le refoulement originaire. Il est certes un processus qui a lieu pour chaque individu de l'espèce, mais il se produit hors de toute intentionnalité de l'individu, il est une opération constitutive et constitutionnelle qui se déroule en dehors de toute participation de l'individu. Celui-ci est non seulement le lieu où elle s'accomplit, mais il en est surtout le résultat, c'est, en effet, par le refoulement originaire que s'amorce le procès d'individuation lui-même. Les refoulements secondaires sont corrélés à l'histoire des contingences d'une vie, le refoulement originaire à l'histoire de l'espèce. Les modalités du refoulement organique ont probablement originairement ${ }^{13}$ davantage de ressemblance avec celles du refoulement originaire qu'avec celles des secondaires, ce n’est qu'ultérieurement qu'elles acquerront les qualités de ces derniers.

Le refoulement dont nous parle cette lettre est un mécanisme normal, il frappe tout individu et est une condition et de son processus d'individuation et de son entrée dans le procès de civilisation. Le quantum de pulsion rendu libre par le refoulement qui conduit à l'abandon de l'érogénéité de la zone

13. C'est sans trop d'hésitation que l'on peut soutenir cela par rapport au passage en arrière-plan, du point de vue d'une hiérarchie des sens, de l'odorat et de la préhension bucco-pharyngienne au profit d'un primat visuo-tactile. Ce passage relève de l'évolution de l'espèce et s'il survient, certes, dans chaque histoire individuelle, il n'en relève pas directement. 
du corps qui constituait antérieurement le lieu d'un investissement libidinal peut, en restant libre, accentuer les processus de déliaison et conduire jusqu'à l'angoisse ou, par établissement d'une nouvelle liaison à la suite d'un retournement en son contraire, il peut s'exprimer dans le rejet. Ce rejet, au travers des formations réactionnelles qui vont l'exprimer sera le «fondement affectif d'une quantité de processus intellectuels du développement, comme la morale, la pudeur, etc. Tout cela apparaît au prix d'une sexualité disparue (virtuelle)».

Nous retrouvons, ici, la logique qui était à l'œuvre dans Caractère et érotisme anal : l'aptitude érogène d'un organe est « absorbée » dans un processus de transposition de l'érotisme d'organe en diverses productions psychiques et, entre autres, en traits de caractère. Le trait de caractère (piété, pudeur, propreté, avarice, générosité, scrupulosité, ambition, etc.) s’exonde de la sexualité disparue, engloutie dans le refoulement mais ce n'est que manifestement que "plus rien des faiblesses et des singularités [de cette sexualité] ne peut être retrouvé », car, derrière le refoulement, l'essentiel reste actuel, la propriété de l'érogénéité « disparue » reste en puissance, riche des nombreuses virtualités que lui offriront les hasards de la vie dont se nourrit l'activité psychique.

Encore un mot à propos de la lettre du 14 novembre 1897. Dans sa réflexion sur ce qu'apporte ce refoulement normal où intervient toujours quelque chose d'organique, Freud souligne que la différence anatomique des sexes qui se traduit par les différences morphologiques entre les organes des corps de la femme et de l'homme a des conséquences sur le processus des refoulements organiques. Pénis, vagin, clitoris (et il faudrait rajouter à cette liste la plupart des caractères sexuels secondaires propre à l'un et à l'autre sexe) ne conservent pas uniformément leur valence érogène, les opérations de retournement en contraire des sensations qui y naissent sont déterminées par les modalités psychiques, historiques et culturelles tant de l'entrée dans le complexe d'Edipe que de son déclin ou de son maintien. Freud souligne que les refoulements organiques « ne se présenteront pas dans le même ordre chronologique selon qu'il s'agit du sexe masculin ou féminin ».

C'est dans « la modification par laquelle la sensation de besoin interne doit se transformer en sensation de dégoût » que réside qu'il puisse y avoir ou non une " utilisation psychique » de la sensation, lorsqu'il y a dégoût « cette utilisation ne livre que des symptômes, au lieu de représentations de but ». Il faut ranger les traits de caractères parmi ce qui est, ici, désigné comme symptômes ${ }^{14}$.

14. Pour la distinction à faire entre le symptôme et le trait de caractère, je renvoie à Villa, 2010. 
POUR CLORE

Je ferai deux remarques pour terminer.

La première concerne la corrélation ironique faite par Voltaire entre changement du corps et changement du caractère. Le refoulement organique en tant que processus pouvant survenir tout au long de la vie, à l'occasion des moments catastrophiques qui ne peuvent que la jalonner, apparaît comme un mécanisme permettant les remaniements psychiques de la représentation du corps qui se traduisent effectivement par un changement du corps et qui laissent ouvertes la voie à de possibles changements du caractère (il nous suffit de penser aux modifications profondes et notables du caractère qui peuvent survenir à l'occasion d'une maladie grave ou d'une rencontre amoureuse).

Ma seconde remarque portera sur le processus de transposition. Nous trouvons l'une des premières occurrences de cette notion dans Les études sur l'hystérie. C'est à propos de l'introduction de la notion de conversion qu'elle survient : la conversion est une " transposition de l'excitation psychique en symptômes durables corporels ». Freud avance que, quand le montant de la conversion est faible, "l'excitation originellement psychique demeur[e] aussi, la plupart du temps, dans le domaine psychique, et il est facile de s'apercevoir que ce cas devient, par là, semblable à ces autres névroses non hystériques ». Par contre, lorsque « la conversion concerne le surcroît de stimulus tout entier, [...] les symptômes corporels de l'hystérie font saillie dans une conscience pleinement normale ». Il est précisé que le plus habituel est que la transposition soit incomplète et que, dans ce cas, une partie au moins du trauma accompagnant l'affect demeure dans la conscience comme composante de l'humeur. Nous ne ferons qu'indiquer que, dans les pages qui suivent cette remarque, bien des notations portent sur des traits et des comportements liés à la question du caractère. Ce sur quoi nous voulions attirer l'attention, c'est que l'absorption de l'érotisme d'organe dans la formation de caractère semble constituer un pendant du processus de conversion. Il y aurait un travail de transposition qui aurait pour effet non pas une modification directe du moi-corps (création d'une zone hystérogène) mais une modification du moi qui se verrait augmenter d'un caractère nouveau : conversion d'un investissement libidinal d'organe en investissement narcissique d'un trait caractériel.

Une fois encore, je conclurai en disant que l'impossibilité de parvenir à une analyse satisfaisante de la formation du caractère est isomorphe à la difficulté que nous rencontrons à dépasser, dans et par la psychanalyse, la résistance que le caractère oppose au traitement psychique.

François Villa 30 bd de Strasbourg 75010 Paris villa@univ-paris-diderot.fr 
BIBLIOGRAPHIE FREUDIENNE SUR LE REFOULEMENT ORGANIQUE

Pour mémoire, voici les différentes occurrences de la notion dans l'œuvre freudienne, lorsque la publication du texte a été posthume, je l'indique.

On trouve des prémisses de la notion dans les lettres du 6-12-1896 et du 12-1-1897, Freud S., Lettres à Wilhem Fließ. 1887-1904, trad. fr. F. Kahn, F. Robert, Paris, Puf, 2006 (Posthume). Première présentation de la notion dans la lettre du 14-11-1897.

Freud S. (1906a), " Mes vues sur le rôle de la sexualité dans l'étiologie des névroses », trad. fr. J. Altounian, A. et O. Bourguignon, G. Goran, J. Laplanche et A. Rauzy, in Résultats, idées, problèmes, t. I, Paris, Puf, 1984. Freud renvoie le lecteur à cette notion comme si elle était évidente, alors qu'il s’agit de sa première mention publique.

Séance du 17 novembre 1909, Les premiers psychanalystes. Minutes de la société de Vienne 1906-1918, t. II, trad. fr. N. Bakman, Paris, Gallimard, 1978, (Posthume).

Freud S. (1909d), « Remarques sur un cas de névrose obsessionnelle. (L’homme aux rats) », trad. fr. M. Bonaparte, R. M. Lœwenstein, in Cinq psychanalyses, Paris, Puf, 1977. Pour Sulloway P. (Freud, biologiste de l'esprit, trad. fr. J. Lelaidier, Paris, Fayard, 1981) c'est la première référence publiée au refoulement organique, là aussi la notion semble aller de soi.

C’est dans Freud S. (1930a), « Le malaise dans la culture », trad. fr. P. Cotet, R. Lainé, J. Stute-Cadiot, in OCF-P, t. XVIII, Paris, Puf, 1994, note 1, p. 286-287 et note 2, p. 292-293, qu'est faite la présentation publique la plus conséquente.

Notons, par ailleurs, que sans être nommé, le mécanisme du refoulement organique est évoqué dans Freud S. :

a) (1912d), «Sur le plus général des rabaissements de la vie amoureuse », trad. fr. D. Berger, J. Laplanche, in La vie sexuelle, Paris, Puf, 1977.

b) (1913k), « Préface à L'ordure dans les mœurs, les usages, les croyances et le droit coutumier » de J.-G. Bourke, trad. fr. P. Cotet, in OCF-P, t. XII, Paris, Puf, 2005.

c) (1919e), « Un enfant est battu », trad. fr. J. Altounian, P. Cotet, in OCF-P, t. XV, Paris, Puf, 1996,

d) (1933b), « Pourquoi la guerre ? », trad. fr. J. Altounian, A. Bourguignon, P. Cotet, A. Rauzy, in OCF-P, t. XIX, Paris, Puf, 1995.

e) (1933a), « Nouvelle suite des leçons d'introduction à la psychanalyse. XXXVé Leçon : D’une vision du monde », trad. fr. J. Altounian, A. Bourguignon, P. Cotet, A. Rauzy, R.-M. Zeitlin, in OCF-P, t. XIX, Paris, Puf, 1995 p. 264-265.

\section{RÉFÉRENCES BIBLIOGRAPHIQUES}

Fédida P., Par où commence le corps humain. Retour sur la régression, Paris, Puf, 2000. Freud S. (1950c [1895]), Projet d'une psychologie, Lettres à Wilhem Fließ. 1887-1904, trad. fr. F. Kahn, F. Robert, Paris, Puf, 2006.

Freud S. (1898), La sexualité dans l'étiologie des névroses, trad. fr J. Altounian, A. Bourguignon, P. Cotet et A. Rauzy, OCF-P, III, Paris, Puf, 1989. 
Freud S. (1905), Trois essais sur la théorie sexuelle, trad. fr. P. Cotet, F. Rexand-Galais, OCF-P, VI, 1901-1906, Paris, Puf, 2006.

Freud S. (1908), Caractère et érotisme anal, trad. fr. F. Kahn, F. Robert, OCF-P, VIII, 1906-1908, Paris, Puf, 2007.

Freud S. (1913-1914[1914c]), Pour introduire le narcissisme, trad. fr. J. Laplanche, OCF-P, XII, Paris, Puf, 2005.

Freud S. [1915 ?(1916-1917e)], « Des transpositions pulsionnelles en particulier dans l'érotisme anal », trad. fr. J. Altounian, A. Bourguignon, P. Cotet, J. Stute-Cadiot, in OCF-P, XV, Paris, Puf, 1996.

Freud S. (1930), Le malaise dans la culture, trad. fr. P. Cotet, R. Lainé, J. Stute-Cadiot, in OCF-P, XVIII, Paris, Puf, 1994.

Villa F., La notion de caractère chez Freud, Paris, Puf, 2009.

Villa F., La puissance de vieillir, Paris, Puf, 2010.

Voltaire, article " Caractère », in Dictionnaire philosophique et compléments, édition de 1765, Paris, cédérom Redon. 